\title{
Ages and metallicities of star clusters: New calibrations and diagnostic diagrams from visible integrated spectra
}

\author{
J. F. C. Santos Jr. ${ }^{1}$ and A. E. Piatti ${ }^{2}$ \\ 1 Departamento de Física, ICEx, UFMG, CP 702, 30123-970 Belo Horizonte, MG, Brazil \\ e-mail: jsantos@fisica.ufmg.br \\ 2 Instituto de Astronomía y Fisica del Espacio, CC 67, Suc. 28, 1428, Buenos Aires, Argentina
}

Received 30 June 2004 / Accepted 28 July 2004

\begin{abstract}
We present homogeneous scales of ages and metallicities for star clusters from very young objects, through intermediate-age ones up to the oldest known clusters. All the selected clusters have integrated spectra in the visible range, as well as reliable determinations of their ages and metallicities. From these spectra equivalent widths ( $E W \mathrm{~s})$ of $\mathrm{KCa}$ II, $\mathrm{G}$ band $(\mathrm{CH})$ and $\mathrm{Mg}$ I metallic, and $\mathrm{H} \delta, \mathrm{H} \gamma$ and $\mathrm{H} \beta$ Balmer lines have been measured homogeneously. The analysis of these $E W$ s shows that the $E W$ sums of the metallic and Balmer $\mathrm{H}$ lines, separately, are good indicators of cluster age for objects younger than $10 \mathrm{Gyr}$, and that the former is also sensitive to cluster metallicity for ages greater than 10 Gyr. We propose an iterative procedure for estimating cluster ages by employing two new diagnostic diagrams and age calibrations based on the above $E W$ sums. For clusters older than $10 \mathrm{Gyr}$, we also provide a calibration to derive their overall metal contents.
\end{abstract}

Key words. galaxies: star clusters - stars: fundamental parameters - techniques: spectroscopic

\section{Introduction}

The determination of age and metallicity of open and globular clusters have contributed to the present knowledge of the structure and chemical evolution of our Galaxy. Properties of star cluster systems in external galaxies, including both Magellanic Clouds (MCs), are also good tracers of the galaxy's chemical enrichment history.

Age determinations of star clusters are frequently based on isochrone matching to color-magnitude diagrams whenever individual star photometry is possible, this technique thus being constrained to spatially resolved objects. Direct metallicity determinations from spectroscopic observations of individual cluster stars also suffer from spatial resolution limitations and they are generally done for stellar systems in the Local Group. Other approaches to estimate metallicity of clusters rely on the position and shape of evolved red features in their color-magnitude diagrams (e.g. Geisler \& Sarajedini 1999).

Integrated colors and spectra have also been used to rank clusters according to their age and metallicity. In particular, the Gunn system photometric classification by Searle et al. (1980) of star clusters in the MCs was later extended to Johnson $U B V$ photometric system in which age calibrations were obtained (Elson \& Fall 1985; Girardi et al. 1995; Bica et al. 1996). Rabin (1982) analyzed a sample of integrated spectra of red star clusters in the MCs proposing a diagnostic diagram involving the equivalent widths $(E W \mathrm{~s})$ of Balmer lines and that of $\mathrm{KCa}$ II. This diagram discriminated most of the MCs clusters from the Galactic globulars, indicating age (and metallicity to a lesser degree) as the main segregation parameter. The lack of a large sample of clusters with well-determined ages and metallicities prevented Rabin from exploring an empirical calibration of the data, although the diagram provided a means to estimate approximate cluster ages from measured $E W \mathrm{~s}$ in the integrated spectra. Aiming at population synthesis studies, Bica \& Alloin (1986a) gathered a library of star cluster spectra in which age and metallicity trends were searched for by plotting $E W \mathrm{~s}$ of various spectral features against age and metallicity. They show that the $E W$ of each Balmer line is a bivalued function of age with a maximum around $300 \mathrm{Myr}$ and that the EWs of prominent metallic features are metallicity indicators for old clusters. Their original sample, composed of 19 MC clusters, 41 Galactic globulars and 3 open clusters, has been substantially expanded over the years in both spectral range and number of objects (Santos et al. 2002; Piatti et al. 2002a, and references therein), and is available by internet (http://vizier.u-strasbg.fr/ cgi-bin/VizieR?-source=III/219).

Recently, a large number of extragalactic star cluster systems has been investigated thanks to the increasing sensitivity of observational technology (see West et al. 2004, and references therein). Even so, only integrated light can be gathered for the more distant systems. By means of such studies it has been possible to examine the origin and evolution of the parent galaxy, as well as to learn about our own Galaxy in a comparative way. On the other hand, the calibration of fundamental 
properties of our local cluster systems against measured indices on integrated light observations is a crucial step to get information on extragalactic cluster systems.

The present work is intended to provide a useful tool for diagnosing the age and the metallicity of single burst stellar populations. The method rests on the measurement of $E W \mathrm{~s}$ in the integrated spectra of star clusters and on the parameters (age and metallicity) taken from the literature put in homogeneous scales. The large dataset for which age and metallicity estimates are available make this a worthwhile analysis. This paper is structured as follows. In Sect. 2 the database is presented, while in Sect. 3 the $E W$ measurements for selected features are detailed. The calibration of the $E W \mathrm{~s}$ as a function of age and metallicity is described in Sect. 4 and the diagnostic diagrams are discussed in Sect. 5. Concluding remarks are given in Sect. 6.

\section{Homogeneous scales of age and metallicity}

The sample selection was based on the availability of cluster integrated spectra with estimated ages and metallicities. Aiming at homogeneity of the sample properties, ages and metallicities were transformed towards a uniform scale, starting with the Galactic globular clusters (GGCs). These procedures have already been used to discuss a possible reorganization of the original template spectra of Bica (1988), by grouping GGCs in different bins, using as criteria, in addition to the metallicity, the age and the horizontal branch morphology (Santos et al. 2002).

\subsection{The galactic globular cluster metallicity scale}

The metallicity scale defined by Carretta \& Gratton (1997, hereafter CG97) was preferred over the widely used Zinn \& West (1984, hereafter ZW84) scale, because the former is based on high-dispersion CCD spectra of giants in 24 clusters, characterizing an homogeneous sample. The metallicities presented by Rutledge et al. (1997, hereafter R97), which were obtained from the near-infrared Ca II triplet ( $\lambda 8498,8542,8662 \AA$ ), were adopted in the present study. Twenty-three globular clusters in our sample are not in R97's sample and their metallicities were taken from ZW84 and transformed to CG97's metallicity scale. The exceptions were metal-rich clusters (see below) and NGC 6540, not present in either sample, for which the quoted metallicity in the Harris (1996) compilation was adopted (from the 2003 updated version of the catalogue available at http: // physwww. physics.mcmaster. ca/ harris/mwgc.dat).

Concerning clusters for which there are metallicities available in the ZW84 scale, their $[\mathrm{Fe} / \mathrm{H}]$ were transformed to the CG97 scale by using Eq. (7) in CG97:

$[\mathrm{Fe} / \mathrm{H}]_{\mathrm{CG}}=a+b[\mathrm{Fe} / \mathrm{H}]_{\mathrm{ZW}}+c[\mathrm{Fe} / \mathrm{H}]_{\mathrm{ZW}}^{2}$

where $a=-0.618 \pm 0.083, b=-0.097 \pm 0.189$, $c=-0.352 \pm 0.067$ and $\sigma\left([\mathrm{Fe} / \mathrm{H}]_{\mathrm{CG}}\right)=0.08$ for 24 clusters. The relationship is valid in the range $-2.24<[\mathrm{Fe} / \mathrm{H}]_{\mathrm{ZW}}<-0.51$ and therefore it was not applied to the metal-rich globular clusters. The metallicity errors were propagated from their original ZW84 values for individual clusters.

\subsubsection{The metal-rich GGCs}

There are discrepancies (larger than $\approx 0.3$ dex) between the aforementioned metallicity scales for the metal-rich clusters $\left([\mathrm{Fe} / \mathrm{H}]_{\mathrm{ZW}}>-0.5\right)$. Specifically, the metallicities of NGC 6316, NGC 6440, NGC 6528, NGC 6553, NGC 6624 and NGC 6637 were reanalyzed in terms of more recent studies in the literature, a task whose fundamental issue was to keep the final adopted metallicities as consistent as possible. The $[\mathrm{Fe} / \mathrm{H}]$ discrepancies are probably produced by uncertainties in the $E W \mathrm{~s}$, since high line crowding in red giant spectra can affect the continuum placement.

Cohen et al. (1999) obtained high-resolution near-infrared spectra of 5 horizontal branch (HB) stars in NGC 6553, and estimated a metallicity of $[\mathrm{Fe} / \mathrm{H}]_{\mathrm{CG}}=-0.16 \pm 0.08$ from uncrowded individual spectral lines, which is much higher than the value quoted by $\mathrm{R} 97\left([\mathrm{Fe} / \mathrm{H}]_{\mathrm{CG}}=-0.60 \pm 0.04\right)$. Later, Carretta et al. (2001) revised Cohen et al.'s estimate to $[\mathrm{Fe} / \mathrm{H}]_{\mathrm{CG}}=-0.06 \pm 0.15$. We adopted this last estimate. Having as one of their goals to extend the calibration of the CG97 metallicity scale to the metal-rich regime, Carretta et al. (2001) obtained $[\mathrm{Fe} / \mathrm{H}]_{\mathrm{CG}}=0.07 \pm 0.10$ for NGC 6528 from high-resolution spectra of $4 \mathrm{HB}$ member stars. They propose a new calibration to transform the ZW84 scale to the CG97 one in order to account for the metal-rich clusters. We have not used such a new calibration because there still seems to remain important discrepancies in the metal-rich regime. For instance, if one assumes $[\mathrm{Fe} / \mathrm{H}]_{\mathrm{ZW}}=0.12 \pm 0.21$ for $\mathrm{NGC} 6528$ and uses this new calibration (Eq. (3) in Carretta et al. 2001), it gives $[\mathrm{Fe} / \mathrm{H}]_{\mathrm{CG}}=1.1 \pm 0.7$. Instead, metallicities for NGC 6528 and NGC 6553 were adopted from the direct measurements on high-dispersion spectra according to the analyses in the literature mentioned above.

NGC $6316\left([\mathrm{Fe} / \mathrm{H}]_{\mathrm{ZW}}=-0.47 \pm 0.15\right)$ and NGC 6440 $\left([\mathrm{Fe} / \mathrm{H}]_{\mathrm{ZW}}=-0.26 \pm 0.15\right)$ have their metallicities revised to lower values $\left([\mathrm{Fe} / \mathrm{H}]_{\mathrm{ZW}}=-0.55 \pm 0.11\right.$ and $[\mathrm{Fe} / \mathrm{H}]_{\mathrm{ZW}}=-0.34 \pm 0.11$, respectively, according to the Armandroff \& Zinn (1988) analysis of integrated nearinfrared spectra). Subsequently, Minniti (1995) determined $[\mathrm{Fe} / \mathrm{H}]_{\mathrm{ZW}}=-0.50 \pm 0.20$ for NGC 6440 based on the spectra of 11 cluster giants together with their near-infrared colors. The adopted metallicity for this cluster was obtained by entering this value into Eq. (1) giving $[\mathrm{Fe} / \mathrm{H}]_{\mathrm{CG}}=-0.66 \pm 0.14$, from a slight extrapolation. In the case of NGC 6316, the mean metallicity from ZW84 and Armandroff \& Zinn (1988) values $\left([\mathrm{Fe} / \mathrm{H}]_{\mathrm{ZW}}=-0.51 \pm 0.19\right)$ transformed to $\mathrm{CG} 97$ scale by using Eq. (1) $\left([\mathrm{Fe} / \mathrm{H}]_{\mathrm{CG}}=-0.66 \pm 0.14\right)$ was adopted in the following analysis.

Heasley et al. (2000) have obtained spectra in the Ca II triplet region of 4 members of NGC 6624 and 7 members of NGC 6637 , and estimated their $[\mathrm{Fe} / \mathrm{H}]_{\mathrm{ZW}}$. These values were transformed to $[\mathrm{Fe} / \mathrm{H}]_{\mathrm{CG}}$ according to Eq. (1) and compared to the ones given by R97. Within the errors, the metallicities are similar: for $\mathrm{NGC} 6624,[\mathrm{Fe} / \mathrm{H}]_{\mathrm{CG}}=-0.70 \pm 0.09$ according 
to Heasley et al. (2000), and $-0.70 \pm 0.03$ following R97; for NGC 6637 the values are $-0.70 \pm 0.09$ and $-0.78 \pm 0.03$, respectively.

\subsection{The galactic globular cluster age scale}

The Galactic globular cluster relative age calibration by Rosenberg et al. (1999, hereafter R99), based on uniform VI CCD color-magnitude diagrams and different sets of isochrones, was adopted. Absolute ages were established with the post-Hipparcos calibration by Carretta et al. (2000), yielding an average absolute age of $13.2 \mathrm{Gyr}$. An uncertainty of $0.1 \mathrm{dex}$ in $[\mathrm{Fe} / \mathrm{H}]$ corresponds to an age precision within 0.25 Gyr (R99). Since just 9 out of 42 globular clusters in our sample have ages in the R99 scale, an age-metallicity relation has been used in order to get ages for 25 clusters with $[\mathrm{Fe} / \mathrm{H}] \leq-0.7$ dex. Specifically, a 2 nd order polinomial was fitted to age as a function of metallicity in the CG97 scale for the 35 clusters in R99 sample, resulting in:

$$
\begin{aligned}
t(\mathrm{Gyr})= & 4.3( \pm 2.2)-11.0( \pm 3.3)[\mathrm{Fe} / \mathrm{H}]_{\mathrm{CG}} \\
& -3.4( \pm 1.1)[\mathrm{Fe} / \mathrm{H}]_{\mathrm{CG}}^{2} .
\end{aligned}
$$

For a given cluster $[\mathrm{Fe} / \mathrm{H}]_{\mathrm{CG}}$, the cluster age and its error were estimated from Eq. (2) and its dispersion at that metallicity, respectively. For the metal-rich clusters with $[\mathrm{Fe} / \mathrm{H}]_{\mathrm{CG}}>-0.7$ we assigned ages of $10.0 \pm 2.0 \mathrm{Gyr}$, which correspond to an extrapolated value.

Table 1 presents the final adopted parameters for the Galactic globular clusters with observed integrated spectra.

\subsection{The galactic open and Magellanic Cloud clusters}

Metallicities and ages were assigned to Galactic open (GOCs) and Magellanic Cloud clusters (MCCs) with observed spectra available. In order to check how smooth the link is between the properties of the oldest clusters in different environments and those of intermediate-age/young clusters, the scales adopted for the younger GGCs were considered. Indeed, NGC 1466 and NGC 6253 allowed us to perform such a comparison. Inevitably, a large number of works on the determination of GOC and MCC properties are based on different observational techniques and methods of analysis. Our attempt to homogenize the cluster properties gathered from the literature is in the hope that the relationship between the observational quantities and the fundamental properties of star systems (e.g., age and metallicity) is not degraded by the lack of consistency among these properties.

For a given cluster, the comparison between ages and/or metallicities estimated from different methods guided us to achieve the final homogeneous dataset. The general guidelines applied for adopting the final parameters were as follows: metallicities obtained from spectroscopic methods were preferred over photometric ones, and whenever the case, its mean value was brought to the same GCC scale according to Eq. (1). Isochrone matching to CMDs and spectral flux distributions were the methods selected in the literature for deciding the final adopted ages. The final adopted parameters of Galactic

\begin{tabular}{|c|c|c|c|c|c|c|}
\hline Cluster & {$[\mathrm{Fe} / \mathrm{H}]_{\mathrm{CG}}$} & $\sigma([\mathrm{Fe} / \mathrm{H}])$ & Ref. & $t(\mathrm{Gyr})$ & $\sigma(t)$ & Ref. \\
\hline \multicolumn{7}{|l|}{ Globular } \\
\hline NGC 104 & -0.78 & 0.02 & 1 & 11.9 & 1.0 & 7 \\
\hline NGC 362 & -1.09 & 0.03 & 1 & 10.2 & 1.0 & 7 \\
\hline NGC 1851 & -1.03 & 0.06 & 1 & 10.6 & 0.9 & 7 \\
\hline NGC 1904 & -1.37 & 0.05 & 1 & 13.2 & 1.1 & 7 \\
\hline NGC 2808 & -1.11 & 0.03 & 1 & 10.7 & 0.9 & 7 \\
\hline NGC 4590 & -2.00 & 0.03 & 1 & 12.4 & 1.1 & 7 \\
\hline NGC 4833 & -1.71 & 0.03 & 1 & 13.4 & 1.0 & 8 \\
\hline NGC 5024 & -1.88 & 0.49 & 2 & 13.2 & 1.0 & 8 \\
\hline NGC 5824 & -1.67 & 0.47 & 2 & 13.4 & 1.0 & 8 \\
\hline NGC 5927 & -0.64 & 0.02 & 1 & 10.0 & 2.0 & 9 \\
\hline NGC 5946 & -1.15 & 0.33 & 2 & 12.5 & 2.0 & 8 \\
\hline NGC 6093 & -1.47 & 0.04 & 1 & 13.7 & 0.9 & 7 \\
\hline NGC 6139 & -1.42 & 0.40 & 2 & 13.2 & 1.0 & 8 \\
\hline NGC 6171 & -0.95 & 0.04 & 1 & 13.5 & 0.9 & 7 \\
\hline NGC 6287 & -1.90 & 0.53 & 2 & 13.2 & 1.0 & 8 \\
\hline NGC 6293 & -1.73 & 0.48 & 2 & 13.4 & 1.0 & 8 \\
\hline NGC 6304 & -0.66 & 0.03 & 1 & 10.0 & 2.0 & 9 \\
\hline NGC 6316 & -0.66 & 0.14 & 2,3 & 10.0 & 2.0 & 9 \\
\hline NGC 6356 & -0.69 & 0.16 & 2 & 10.0 & 2.0 & 9 \\
\hline NGC 6388 & -0.74 & 0.18 & 2 & 10.6 & 2.0 & 8 \\
\hline NGC 6401 & -0.96 & 0.27 & 2 & 11.8 & 2.0 & 8 \\
\hline NGC 6402 & -1.16 & 0.32 & 2 & 12.6 & 2.0 & 8 \\
\hline NGC 6440 & -0.66 & 0.14 & 4 & 10.0 & 2.0 & 9 \\
\hline NGC 6453 & -1.29 & 0.37 & 2 & 12.9 & 1.0 & 8 \\
\hline NGC 6517 & -1.12 & 0.32 & 2 & 12.5 & 2.0 & 8 \\
\hline NGC 6528 & 0.07 & 0.10 & 5 & 10.0 & 2.0 & 9 \\
\hline NGC 6540 & -1.2 & 0.5 & 6 & 12.7 & 2.0 & 8 \\
\hline NGC 6541 & -1.53 & 0.03 & 1 & 13.3 & 1.0 & 8 \\
\hline NGC 6544 & -1.20 & 0.04 & 2 & 12.7 & 1.5 & 8 \\
\hline NGC 6553 & -0.06 & 0.15 & 5 & 10.0 & 2.0 & 9 \\
\hline NGC 6558 & -1.21 & 0.34 & 2 & 12.7 & 1.5 & 8 \\
\hline NGC 6569 & -0.79 & 0.20 & 2 & 10.9 & 2.0 & 8 \\
\hline NGC 6624 & -0.70 & 0.03 & 1 & 10.4 & 1.5 & 8 \\
\hline NGC 6637 & -0.78 & 0.03 & 1 & 10.9 & 2.0 & 8 \\
\hline NGC 6638 & -0.90 & 0.04 & 1 & 11.5 & 2.0 & 8 \\
\hline NGC 6642 & -1.08 & 0.31 & 2 & 12.3 & 2.0 & 8 \\
\hline NGC 6652 & -0.81 & 0.21 & 2 & 11.1 & 2.0 & 8 \\
\hline NGC 6715 & -1.25 & 0.07 & 1 & 12.9 & 1.5 & 8 \\
\hline NGC 6760 & -0.66 & 0.14 & 2 & 10.0 & 2.0 & 9 \\
\hline NGC 6864 & -1.10 & 0.30 & 2 & 12.4 & 2.0 & 8 \\
\hline NGC 7006 & -1.35 & 0.36 & 2 & 13.1 & 1.0 & 8 \\
\hline NGC 7078 & -2.02 & 0.04 & 1 & 12.9 & 0.6 & 7 \\
\hline
\end{tabular}

Table 1. Homogeneous ages and metallicities: Milky Way clusters.

open and Magellanic Cloud clusters with observed spectra are given in Tables 1 and 2, respectively. Below, we describe the intercomparison between different studies for clusters deserving some comments, which illustrates the process of the parameter merging employed.

The metallicity of NGC 6253 derived by Twarog et al. (2003) using Strömgren photometric indices and $\mathrm{Ca}$ and 
Table 1. continued.

\begin{tabular}{|c|c|c|c|c|c|c|}
\hline Cluster & {$[\mathrm{Fe} / \mathrm{H}]_{\mathrm{CG}}$} & $\sigma([\mathrm{Fe} / \mathrm{H}])$ & Ref. & $\overline{t(\mathrm{Gyr})}$ & $\sigma(t)$ & Ref. \\
\hline \multicolumn{7}{|l|}{ Open } \\
\hline NGC 2158 & -0.25 & 0.09 & 10 & 2.0 & 0.5 & 17 \\
\hline vdB-RN 80 & 0.0 & 0.2 & 11 & 0.0045 & 0.0015 & 16 \\
\hline NGC 2368 & 0.0 & 0.2 & 11 & 0.05 & 0.01 & 16 \\
\hline Berkeley 75 & 0.0 & 0.2 & 11 & 3.0 & 1.0 & 16 \\
\hline Haffner 7 & 0.0 & 0.2 & 11 & 0.10 & 0.01 & 16 \\
\hline ESO 429-SC13 & 0.0 & 0.2 & 11 & 0.10 & 0.05 & 16 \\
\hline NGC 2660 & -0.18 & 0.06 & 12 & 1.1 & 0.1 & 16 \\
\hline UKS 2 & 0.0 & 0.2 & 11 & 0.8 & 0.2 & 16 \\
\hline Ruprecht 83 & 0.0 & 0.2 & 11 & 0.055 & 0.020 & 16 \\
\hline Hogg 3 & 0.0 & 0.2 & 11 & 0.075 & 0.025 & 16 \\
\hline NGC 3293 & 0.0 & 0.2 & 11 & 0.006 & 0.001 & 16 \\
\hline Bochum 12 & 0.0 & 0.2 & 11 & 0.045 & 0.015 & 16 \\
\hline Pismis 17 & 0.0 & 0.2 & 11 & 0.0045 & 0.0015 & 16 \\
\hline Hogg 11 & 0.0 & 0.2 & 11 & 0.008 & 0.005 & 16 \\
\hline ESO 93-SC08 & -0.4 & 0.2 & 13 & 5.5 & 1.0 & 13 \\
\hline MEL 105 & 0.00 & 0.25 & 14 & 0.3 & 0.05 & 16 \\
\hline BH 132 & 0.0 & 0.2 & 11 & 0.15 & 0.05 & 16 \\
\hline Hogg 15 & 0.0 & 0.2 & 11 & 0.02 & 0.01 & 16 \\
\hline Pismis 18 & 0.0 & 0.2 & 11 & 1.2 & 0.4 & 16 \\
\hline NGC 5606 & 0.09 & 0.25 & 14 & 0.006 & 0.002 & 16 \\
\hline NGC 5999 & 0.0 & 0.2 & 11 & 0.3 & 0.1 & 16 \\
\hline NGC 6031 & 0.0 & 0.2 & 11 & 0.2 & 0.1 & 16 \\
\hline Ruprecht 119 & 0.0 & 0.2 & 11 & 0.015 & 0.010 & 16 \\
\hline NGC 6178 & 0.0 & 0.2 & 11 & 0.04 & 0.01 & 16 \\
\hline Lyngå 11 & 0.0 & 0.2 & 11 & 0.45 & 0.05 & 16 \\
\hline NGC 6253 & 0.5 & 0.1 & 15 & 3.0 & 0.5 & 15 \\
\hline BH 217 & 0.0 & 0.2 & 11 & 0.020 & 0.015 & 16 \\
\hline NGC 6318 & 0.0 & 0.2 & 11 & 0.02 & 0.02 & 16 \\
\hline NGC 6520 & -0.25 & 0.25 & 14 & 0.19 & 0.04 & 16 \\
\hline NGC 6603 & 0.0 & 0.2 & 11 & 0.35 & 0.10 & 16 \\
\hline Ruprecht 144 & 0.0 & 0.2 & 11 & 0.15 & 0.05 & 16 \\
\hline NGC 6705 & 0.14 & 0.04 & 12 & 0.25 & 0.05 & 16 \\
\hline NGC 6756 & 0.0 & 0.2 & 11 & 0.3 & 0.1 & 16 \\
\hline
\end{tabular}

References: (1) Rutledge et al. (1997); (2) Zinn \& West (1984) plus Eq. (1); (3) Armandroff \& Zinn (1988) plus Eq. (1); (4) Minniti (1995) plus Eq. (1); (5) Carretta et al. (2001); (6) Harris (1996); (7) Rosenberg et al. (1999); (8) Eq. (2); (9) assumed age for metal-rich GGCs (see Sect. 2.2); (10) Friel et al. (2002); (11) assumed [Fe/H] for open clusters (see Sect. 2.3); (12) Twarog et al. (1997); (13) Phelps \& Schick (2003); (14) Tadross (2003); (15) Twarog et al. (2003); (16) Piatti et al. (2002a); (17) Carraro et al. (2002).

$\mathrm{H}_{\beta}$ filters was adopted in the present work. Its age was also adopted from Twarog et al. (2003), who transformed $b-y$ colors to $B-V$ colors in order to obtain a suitable CMD for isochrone matching (Padova and Geneva models used). We note that Anthony-Twarog \& Twarog (2000) obtained $[\mathrm{Fe} / \mathrm{H}]=-1.82 \pm 0.04$ and age $=12.0 \pm 0.8 \mathrm{Gyr}$ for NGC 6397 using the same kind of data and approach as Twarog et al. (2003). In our adopted scales, NGC 6397 has $[\mathrm{Fe} / \mathrm{H}]=-1.76 \pm$ 0.03 and age $=13.2 \pm 0.9 \mathrm{Gyr}$, which essentially are, within the
Table 2. Homogeneous ages and metallicities: Magellanic Cloud clusters.

\begin{tabular}{lrrrrrr}
\hline \hline Cluster & {$[\mathrm{Fe} / \mathrm{H}]_{\mathrm{CG}}$} & $\sigma([\mathrm{Fe} / \mathrm{H}])$ & Ref. & $t(\mathrm{Gyr})$ & $\sigma(t)$ & Ref. \\
\hline LMC & & & & & & \\
\hline NGC 1466 & -1.64 & 0.49 & 18,37 & 13.1 & 1.5 & 29 \\
NGC 1711 & -0.68 & 0.15 & 19 & 0.068 & 0.009 & 30 \\
NGC 1783 & -0.65 & 0.14 & 20 & 1.3 & 0.4 & 35 \\
NGC 1805 & -0.2 & 0.2 & 21 & 0.014 & 0.006 & 31 \\
NGC 1831 & -0.62 & 0.09 & 23 & 0.32 & 0.12 & 30 \\
NGC 1850 & -0.12 & 0.03 & 22 & 0.031 & 0.009 & 32 \\
NGC 1854 & -0.50 & 0.10 & 22 & 0.034 & 0.008 & 32 \\
NGC 1856 & -0.17 & 0.27 & 25 & 0.151 & 0.040 & 32 \\
NGC 1866 & -0.66 & 0.14 & 24 & 0.15 & 0.05 & 30 \\
NGC 1868 & -0.66 & 0.14 & 18 & 0.85 & 0.11 & 30 \\
NGC 1978 & -0.85 & 0.24 & 24 & 2.2 & 0.4 & 24 \\
NGC 1984 & -0.90 & 0.40 & 26 & 0.004 & 0.004 & 33 \\
NGC 2004 & -0.56 & 0.03 & 22 & 0.028 & 0.018 & 30 \\
NGC 2011 & -0.47 & 0.40 & 26 & 0.005 & 0.001 & 32 \\
NGC 2100 & -0.32 & 0.03 & 22 & 0.032 & 0.019 & 30 \\
\hline SMC & & & & & & \\
\hline NGC 121 & -1.19 & 0.12 & 27 & 11.9 & 1.3 & 36 \\
NGC 330 & -0.82 & 0.10 & 27 & 0.025 & 0.015 & 27 \\
NGC 419 & -0.70 & 0.30 & 27 & 1.2 & 0.5 & 27 \\
K 3 & -0.98 & 0.12 & 27 & 6.0 & 1.3 & 36 \\
K 28 & -1.2 & 0.2 & 28 & 2.1 & 0.5 & 28 \\
\hline
\end{tabular}

References: (18) Olszewski et al. (1991) and Eq. (1); (19) Dirsch et al. (2000) and Eq. (1); (20) Cohen (1982) and Eq. (1); (21) Johnson et al. (2001); (22) Jasniewicz \& Thévenin (1994); (23) Leonardi \& Rose (2003); (24) Hill et al. (2000) and Eq. (1); (25) Beasley et al. (2002); (26) Oliva \& Origlia (1998); (27) Da Costa \& Hatzidimitriou (1998); (28) Piatti et al. (2001); (29) Johnson et al. (1999) (see Sect. 2.3); (30) Girardi et al. (1995); (31) de Grijs et al. (2002); (32) Bica et al. (1990); (33) Santos et al. (1995); (34) Piatti et al. (2002b); (35) Geisler et al. (1997); (36) Mighell et al. (1998); (37) Suntzeff et al. (1992) and Eq. (1).

uncertainties, the same values as obtained by Anthony-Twarog \& Twarog (2000).

For Melotte 105, NGC 5606 and NGC 6520, the metallicities obtained by Tadross (2003) from the $U V$ excess method (Cameron 1985) were adopted, since these clusters lack spectroscopy based estimates. The uncertainty from the original work by Cameron (1985), namely \pm 0.25 in $[\mathrm{Fe} / \mathrm{H}]$, is such that it should encompass different scales.

Concerning MCCs, Olszewski et al. (1991) have determined $[\mathrm{Fe} / \mathrm{H}]_{\mathrm{ZW}}$ for 4 clusters in our sample (NGC 1466, NGC 1831, NGC 1868, NGC 1978), from measurements of Ca II near-infrared triplet $E W \mathrm{~s}$.

Geisler et al. (1997) calibrated the magnitude difference $\delta T_{1}$ between the giant branch clump and the turnoff in terms of age using LMC and Galactic standard clusters older than 1 Gyr. The mean ages of 7 LMC standard clusters older 
than $\approx 10 \mathrm{Gyr}$ is $\langle$ age $(\mathrm{Gyr})\rangle=14.0 \pm 0.9$. Olsen et al. (1998) observed another 6 old LMC clusters and provided relative ages by using the GGCs M 3, M 5 and M 55 as age standards. We computed the mean age of these 6 LMC clusters from the absolute ages of M 3, M 5 and M 55 according to R99 and Carretta et al. (2000) and obtained $\langle\operatorname{age}(\mathrm{Gyr})\rangle=14.0 \pm 1.4$. Since the mean ages of the old objects in the Geisler et al. (1997) and Olsen et al. (1998) samples are in very good agreement, we deduce that, on average, the age scale by Geisler et al. (1997) is compatible with the scale adopted in the present study.

The following analysis of NGC 1466 reinforces such a compatibility. The cluster $[\mathrm{Fe} / \mathrm{H}]_{\mathrm{ZW}}$, as derived by Olszewski et al. (1991) and revised by Suntzeff et al. (1992), aiming at consistency with RR Lyrae analyses, was transformed to $[\mathrm{Fe} / \mathrm{H}]_{\mathrm{CG}}$. Johnson et al. (1999) used this metallicity value and the cluster HST CMD to derive its age. On the basis of isochrone matching, they obtained the same age value as that of M 3 and M92, with an error of $1.5 \mathrm{Gyr}$. Since R99 provide identical ages for these GGCs $(13.1 \mathrm{Gyr})$, we use it for NGC 1466. This age incorporates the value $12.7 \mathrm{Gyr}$ determined by Geisler et al. (1997) from the $\delta T_{1}$ age index.

Geisler et al. (1997) also measured ages for NGC 1783 and NGC 1978. We adopted their age for NGC 1783, with errors that encompass previous works. In the case of NGC 1978, instead, we adopted the age estimated by Testa et al. (1999) from the fit of different sets of isochrones with $[\mathrm{Fe} / \mathrm{H}]_{\mathrm{ZW}}=-0.4$. The metal content assumed by Testa et al. (1999) is compatible with the more recent and reliable determination obtained by Hill et al. (2000). They employed high resolution spectra obtained with the VLT, and determined $[\mathrm{Fe} / \mathrm{H}]_{\mathrm{ZW}}$ for NGC 1978 and NGC 1866. We adopted the estimated metallicities of Hill et al. (2000) for NGC 1978 and NGC 1866 transformed to CG97 scale.

Jasniewicz \& Thévenin (1994) have observed spectra of stars at intermediate resolution in the clusters NGC 1850, NGC 1854, NGC 2004, and NGC 2100, comparing them with synthetic spectra in order to obtain metallicities, which we adopted. One star was observed in NGC 1850 and the error adopted arbitrarily corresponds to $20 \%$.

Girardi et al. (1995) have remeasured age-sensitive indices in the CMDs of NGC 1711, NGC 1831, NGC 1866, NGC 1868, NGC 2004, NGC 2100, NGC 2134, NGC 2164 and NGC 2214, making it an homogeneous sample. We have checked consistency with our scale by comparing the age that they obtained for NGC 1866 with that by Testa et al. (1999) (also estimated by Geisler et al. 1997), finding both values similar $(0.15 \pm 0.06 \mathrm{Gyr}$ and $0.15 \pm 0.05 \mathrm{Gyr}$, respectively $)$. We have used ages by Girardi et al. (1995) for the aforementioned clusters.

Dirsch et al. (2000) determined ages and metallicities for NGC 1711, NGC 2031 and NGC 2136 based on CCD Strömgren photometry. Different isochrone sets were employed to derive ages. Judging by NGC 2031 and NGC 1711 $(0.16 \pm 0.04 \mathrm{Gyr}$ and $0.050 \pm 0.006 \mathrm{Gyr}$, respectively $)$, also in the Girardi et al. (1995) sample, the ages are on the same scale. Their metallicities are in the ZW84 system, and therefore, the estimate for NGC 1711 has been transformed to the CG97 system.
For SMC clusters, the metallicities in the CG scale and homogeneous ages according to Da Costa \& Hatzidimitriou (1998) were adopted, except for K 28 (Piatti et al. 2001) and for NGC 121 and K3, whose ages are from Mighell et al. (1998).

\section{The integrated spectra and equivalent widths}

$E W \mathrm{~s}$ of metallic features (K Ca II, G band $(\mathrm{CH})$ and $\mathrm{Mg}$ I) and Balmer lines $(\mathrm{H} \delta, \mathrm{H} \gamma$ and $\mathrm{H} \beta$ ) were taken from Bica \& Alloin (1986b), except for the LMC clusters NGC 1711, NGC 1805, NGC 1850, NGC 1854, NGC 1984 and NGC 2011, which were taken from Santos et al. (1995). This source provides the $E W$ of $\mathrm{Mg} \mathrm{I}+\mathrm{MgH}$, which is a sum of three adjacent windows. We have measured in those spectra the central window (5156-5196 $\AA$ ), which is the one employed in the present work, according to the definitions in Bica \& Alloin (1986a). $E W$ s measured for NGC 6520 and Mel 105 were taken from Santos \& Bica (1993). EWs measurements of all six windows were carried out for ESO 93-SC08, NGC 5606, NGC 6253, NGC 6540, K 3 and K 28.

We emphasize that the measurement of the $E W \mathrm{~s}$ follows a uniform procedure: first, the continuum placement according to well-determined spectral fluxes is fitted and, second, the spectral windows as defined by Bica \& Alloin (1986a) are fixed. Limits for the K CaII, G band $(\mathrm{CH}), \mathrm{MgI}, \mathrm{H} \delta, \mathrm{H} \gamma$ and $\mathrm{H} \beta$ spectral windows are, respec-

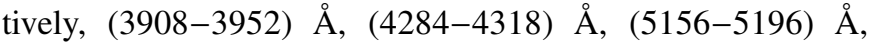

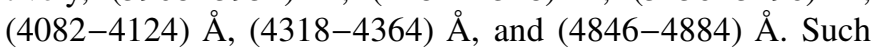
a procedure has been applied consistently for all of the cluster sample, making the $E W \mathrm{~s}$ from integrated spectra safely comparable and useful to study stellar populations in general.

Before measuring the $E W \mathrm{~s}$, the spectra were set to the restframe according to the Doppler shift of $\mathrm{H}$ Balmer lines. Then, the spectra were normalized to $F_{\lambda}=1$ at $5870 \AA$ and smoothed to the typical resolution of the database $(\approx 10-15 \AA)$.

The $E W \mathrm{~s}$ of $\mathrm{H}$ Balmer, KCaII, $\mathrm{G}$ band $(\mathrm{CH})$ and $\operatorname{MgI}(5167+5173+5184 \AA)$ were measured using the IRAF task splot. Tables 3 and 4 present these measurements. Typical errors of $10 \%$ on individual $E W$ measurements were obtained by employing slightly different continua.

$E W s$ of the selected three metallic features have been shown to be well correlated with metallicity for the old GGCs (Bica \& Alloin 1986a). EWs of Balmer lines, being sensitive to the flux of turnoff stars for intermediate-age/young clusters, are expected to change with cluster age, reaching a maximum whenever A-type stars dominate the turnoff (Bica \& Alloin 1986a).

Taking into account these trends and, with the aim of finding spectral indices with a higher sensibility to the cluster ages and metallicities, we analysed the behaviour of the sum of $E W \mathrm{~s}$ of the three metallic lines and the three Balmer lines. As a by-product, the relative errors of these sums resulted in $\approx 7 \%$ smaller errors than the individual spectral window. A similar approach has been shown to be useful in the discrimination of old and intermediate-age/young systems (Rabin 1982; Dutra et al. 1999). 
Table 3. Equivalent widths: Milky Way clusters.

\begin{tabular}{lrrrrrr}
\hline \hline Window & K Ca II & G band CH & Mg I & H $\delta$ & H $\gamma$ & H $\beta$ \\
Cluster & & & & & & \\
\hline Globular & & & & & & \\
\hline NGC 104 & 12.9 & 5.3 & 5.6 & 2.2 & 1.8 & 2.9 \\
NGC 362 & 10.5 & 3.9 & 1.9 & 2.1 & 2.6 & 2.9 \\
NGC 1851 & 10.5 & 3.1 & 2.0 & 4.1 & 3.6 & 3.5 \\
NGC 1904 & 9.1 & 3.2 & 1.2 & 3.7 & 3.5 & 3.4 \\
NGC 2808 & 8.5 & 3.7 & 1.6 & 2.9 & 2.8 & 3.3 \\
NGC 4590 & 4.9 & 1.5 & 1.8 & 3.6 & 3.4 & 3.8 \\
NGC 4833 & 4.1 & 3.7 & 2.2 & 6.4 & 4.6 & 3.8 \\
NGC 5024 & 5.7 & 2.7 & 1.0 & 3.8 & 3.7 & 3.6 \\
NGC 5824 & 5.3 & 2.9 & 1.3 & 4.5 & 4.8 & 2.7 \\
NGC 5927 & 14.6 & 9.0 & 6.2 & 4.7 & 4.9 & 4.1 \\
NGC 5946 & 10.1 & 6.2 & 2.1 & 6.6 & 5.3 & 3.4 \\
NGC 6093 & 5.8 & 3.5 & 1.3 & 4.1 & 4.8 & 3.2 \\
NGC 6139 & 6.1 & 3.8 & 3.3 & 3.8 & 5.4 & 3.9 \\
NGC 6171 & 14.4 & 7.2 & 5.0 & 3.8 & 5.8 & 2.7 \\
NGC 6287 & 5.8 & 3.6 & 1.8 & 4.7 & 4.4 & 3.1 \\
NGC 6293 & 6.4 & 4.2 & 0.9 & 5.9 & 5.8 & 4.9 \\
NGC 6304 & 17.4 & 7.8 & 5.6 & 4.0 & 5.6 & 2.7 \\
NGC 6316 & 15.0 & 8.4 & 5.3 & 2.0 & 5.8 & 2.9 \\
NGC 6356 & 17.5 & 8.1 & 5.1 & 3.5 & 4.2 & 2.8 \\
NGC 6388 & 14.3 & 5.8 & 5.3 & 4.3 & 4.1 & 2.5 \\
NGC 6401 & 11.1 & 6.5 & 4.7 & 9.3 & 10.8 & 4.2 \\
NGC 6402 & 5.4 & 5.9 & 3.2 & 6.0 & 6.7 & 3.9 \\
NGC 6440 & 17.1 & 9.3 & 7.8 & 7.2 & 7.4 & 4.8 \\
NGC 6453 & 6.6 & 4.9 & 1.7 & 4.3 & 4.9 & 3.9 \\
NGC 6864 & 11.2 & 4.2 & 3.2 & 3.2 & 3.3 & 3.8 \\
NGC 6517 & 9.8 & 6.5 & 0.3 & 7.4 & 6.6 & 3.5 \\
NGC 6528 & 15.9 & 8.3 & 8.4 & 5.2 & 6.3 & 4.3 \\
NGC 6540 & 14.8 & 7.5 & 5.3 & 2.7 & 3.7 & 3.2 \\
NGC 6541 & 6.7 & 3.1 & 1.4 & 3.8 & 2.6 & 4.0 \\
NGC 6544 & 8.4 & 4.9 & 3.3 & 3.4 & 10.9 & 4.1 \\
NGC 6553 & 18.6 & 14.0 & 8.5 & 0.4 & 5.4 & 6.2 \\
NGC 6558 & 10.5 & 4.8 & 3.0 & 6.3 & 4.1 & 4.9 \\
NGC 6569 & 13.7 & 7.7 & 5.5 & 5.2 & 6.5 & 2.3 \\
NGC 6624 & 15.5 & 6.3 & 5.4 & 3.6 & 4.2 & 2.5 \\
NGC 6637 & 14.0 & 8.0 & 3.2 & 2.2 & 3.6 & 1.8 \\
NGC 6638 & 12.0 & 5.6 & 3.0 & 3.7 & 4.8 & 4.2 \\
NGC 6642 & 8.5 & 7.7 & 4.4 & 5.4 & 5.7 & 4.3 \\
NGC & 11.4 & 6.4 & 3.5 & 2.9 & 3.6 & 2.7 \\
NGC & 15.1 & 4.9 & 2.8 & 3.6 & 4.4 & 3.0 \\
NG & 2.4 & 0.9 & 3.2 & 4.0 & 2.5 \\
\hline NG & & & & &
\end{tabular}

\section{Calibrating age and $[\mathrm{Fe} / \mathrm{H}]$ using $\Sigma E W(\mathrm{~K}+\mathrm{CH}+\mathrm{Mg})$ and $\Sigma E W(\mathrm{H} \delta+\mathrm{H} \gamma+\mathrm{H} \beta)$}

Figure 1 presents the $E W$ sums against cluster age and metallicity. Different symbols represent clusters of different type or parent galaxy as indicated at the top of the upper-left panel. Error bars are not shown for clarity purposes. At a first glance, both metallic and Balmer line $E W$ sums seem to be correlated with the age of clusters younger than $10 \mathrm{Gyr}$
Table 3. continued.

\begin{tabular}{lrrrrrr}
\hline \hline Window & K Ca II & G band CH & Mg I & H $\delta$ & $\mathrm{H} \gamma$ & $\mathrm{H} \beta$ \\
Cluster & & & & & & \\
\hline Open & & & & & & \\
\hline NGC 2158 & 13.4 & 4.4 & 5.0 & 10.1 & 8.3 & 7.4 \\
vdB-RN 80 & 0.6 & 0.3 & 1.2 & 6.5 & 5.8 & 5.3 \\
NGC 2368 & 3.2 & 4.3 & 2.1 & 10.7 & 10.1 & 8.7 \\
Berkeley 75 & 10.4 & 9.2 & 5.0 & 4.0 & 6.3 & 3.5 \\
Haffner 7 & 1.6 & 4.4 & 3.3 & 8.7 & 15.4 & 7.3 \\
ESO 429-SC13 & 1.3 & 3.4 & 2.6 & 8.7 & 11.6 & 8.7 \\
NGC 2660 & 8.4 & 3.8 & 3.1 & 8.4 & 7.8 & 8.2 \\
UKS 2 & 7.7 & 5.4 & 3.3 & 9.3 & 6.9 & 5.7 \\
Ruprecht 83 & 2.3 & 1.6 & 1.4 & 11.5 & 8.5 & 8.3 \\
Hogg 3 & 5.3 & 1.8 & 1.5 & 8.4 & 8.1 & 7.6 \\
NGC 3293 & -1.5 & 2.1 & 1.0 & 3.0 & 4.4 & 1.2 \\
Bochum 12 & 4.5 & 2.6 & 2.3 & 7.6 & 8.6 & 6.4 \\
Pismis 17 & 2.0 & 2.5 & 0.5 & 5.0 & 6.9 & 1.4 \\
Hogg 11 & 2.7 & -0.2 & 0.3 & 4.5 & 3.5 & 3.6 \\
ESO 93-SC08 & 12.5 & 6.1 & 4.8 & 6.7 & 4.6 & 4.2 \\
MEL 105 & 2.3 & 2.4 & 1.3 & 10.7 & 11.0 & 9.6 \\
BH 132 & 4.6 & 1.1 & 2.9 & 15.1 & 8.6 & 7.2 \\
Hogg 15 & 0.0 & 0.3 & 0.7 & 5.7 & 3.9 & 3.8 \\
Pismis 18 & 6.4 & 4.8 & 2.8 & 7.5 & 6.3 & 6.4 \\
NGC 5606 & 1.5 & 0.4 & 0.5 & 5.1 & 4.3 & 3.6 \\
NGC 5999 & 3.3 & 3.0 & 3.2 & 10.5 & 9.8 & 8.3 \\
NGC 6031 & 2.0 & 1.0 & 0.4 & 9.5 & 8.8 & 7.7 \\
Ruprecht 119 & 0.9 & 1.1 & 1.3 & 6.9 & 5.0 & 3.8 \\
NGC 6178 & 0.5 & 0.2 & 0.2 & 7.6 & 5.5 & 5.3 \\
Lyngå 11 & 5.3 & 3.8 & 2.5 & 9.5 & 10.1 & 7.6 \\
NGC 6253 & 11.8 & 4.9 & 7.4 & 8.7 & 3.0 & 6.4 \\
BH 217 & 2.3 & 2.3 & 0.6 & 6.4 & 5.5 & 3.3 \\
NGC 6318 & 3.7 & 4.6 & 1.0 & 8.8 & 9.8 & 4.8 \\
NGC 6520 & 3.0 & 3.5 & 2.3 & 7.4 & 8.7 & 6.6 \\
NGC 6603 & 5.6 & 3.4 & 2.1 & 14.3 & 14.3 & 12.2 \\
Ruprecht 144 & 1.3 & 2.0 & 0.9 & 9.4 & 11.0 & 7.3 \\
NGC 6705 & 2.9 & 2.6 & 2.1 & 10.5 & 10.1 & 8.6 \\
NGC 6756 & 1.7 & 1.8 & 1.1 & 11.2 & 8.6 & 8.7 \\
\hline & & & & & &
\end{tabular}

(upper- and lower-left panels). According to the lower right panel, the sum of metallic line EWs also appears to be sensitive to the GGC metallicities. However, none of the $E W$ sums is correlated with GGC ages - despite the fact that they cover an appreciable age range $(t \approx 10-14 \mathrm{Gyr})-$, nor with the metallicity of clusters younger than $10 \mathrm{Gyr}$.

According to the observed behaviours, we decided to fit both $E W$ sums in terms of $\log t$ for clusters younger than $10 \mathrm{Gyr}$, and the metallic line $E W$ sum as a function of GGC metal content. Figure 2 shows the same plots as Fig. 1, but including error bars and curve fittings superimposed on diagrams for which a correlation was found. Ranges of age and metallicity were selected as well to highlight their correlations with the $E W \mathrm{~s}$. For the relationship between the $\Sigma E W(\mathrm{H} \delta+\mathrm{H} \gamma+\mathrm{H} \beta)$ and the cluster age (upper-left panel), we 


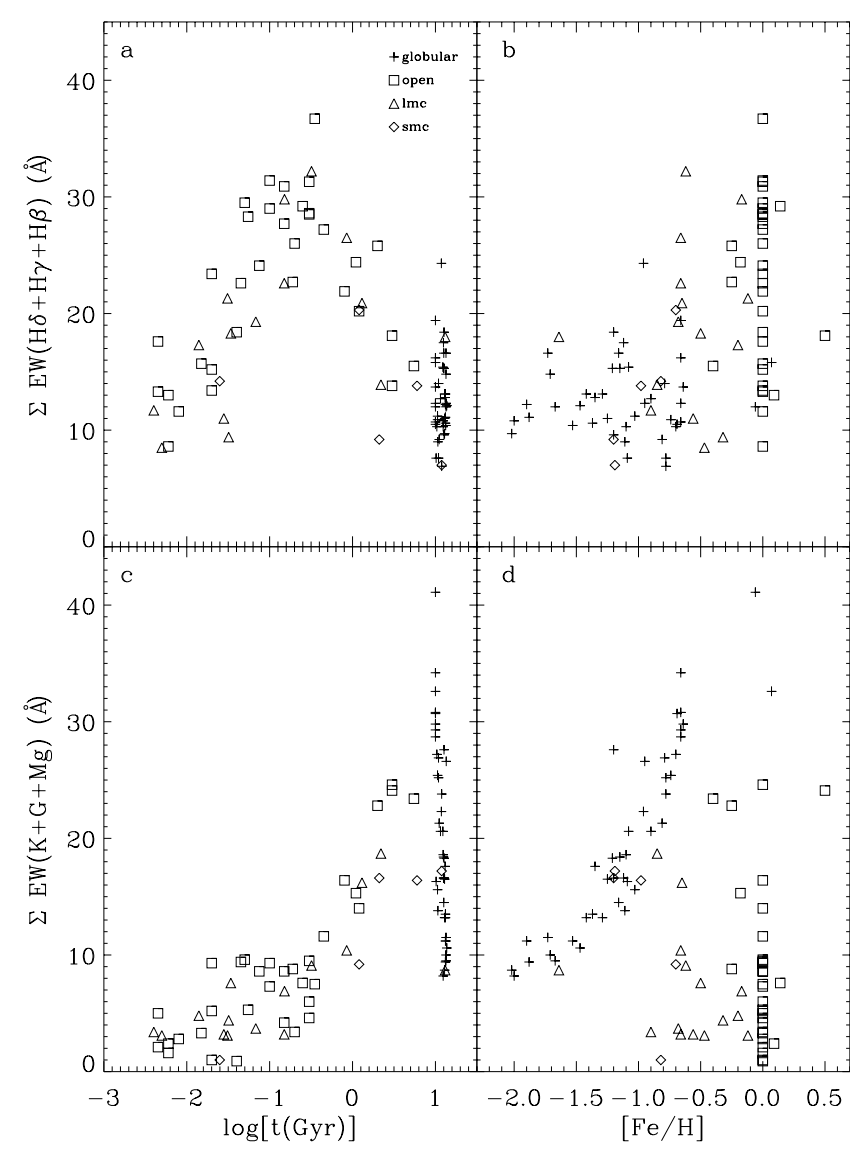

Fig. 1. Sum of metallic and Balmer H equivalent widths against cluster parameters. Different symbols distinguish Milky Way and Magellanic Clouds star clusters.

Table 4. Equivalent widths: Magellanic Cloud clusters.

\begin{tabular}{lrrrrrr}
\hline \hline Window & K Ca II & G band CH & Mg I & $\mathrm{H} \delta$ & $\mathrm{H} \gamma$ & $\mathrm{H} \beta$ \\
Cluster & & & & & & \\
\hline LMC & & & & & & \\
\hline NGC 1466 & 2.6 & 3.1 & 3.0 & 7.0 & 6.5 & 4.5 \\
NGC 1711 & 1.4 & 1.1 & 1.2 & 7.0 & 6.8 & 5.5 \\
NGC 1783 & 8.5 & 4.4 & 3.3 & 7.5 & 6.9 & 6.5 \\
NGC 1805 & 1.0 & 1.3 & 2.5 & 6.6 & 6.0 & 4.7 \\
NGC 1831 & 4.7 & 2.6 & 1.8 & 14.6 & 10.4 & 7.2 \\
NGC 1850 & 1.1 & 1.0 & 1.0 & 7.4 & 7.1 & 6.8 \\
NGC 1854 & 2.9 & 2.2 & 2.5 & 7.8 & 7.3 & 3.2 \\
NGC 1856 & 2.1 & 2.9 & 1.9 & 11.6 & 10.0 & 8.2 \\
NGC 1866 & 0.8 & 1.2 & 1.2 & 8.8 & 6.8 & 7.0 \\
NGC 1868 & 6.3 & 1.4 & 2.7 & 10.9 & 7.7 & 7.9 \\
NGC 1978 & 11.1 & 4.5 & 3.1 & 3.7 & 4.8 & 5.4 \\
NGC 1984 & 0.9 & 1.5 & 1.0 & 4.2 & 5.3 & 2.2 \\
NGC 2004 & 1.2 & 0.0 & 2.0 & 4.3 & 3.2 & 3.5 \\
NGC 2011 & 0.8 & 0.9 & 1.4 & 2.6 & 3.9 & 2.0 \\
NGC 2100 & 2.5 & 0.4 & 1.5 & 4.1 & 3.5 & 1.8 \\
\hline SMC & & & & & & \\
\hline NGC 121 & 11.6 & 3.5 & 2.1 & 2.7 & 1.2 & 3.1 \\
NGC 330 & 0.5 & 0.5 & 0.0 & 5.5 & 4.1 & 4.6 \\
NGC 419 & 4.8 & 3.3 & 1.1 & 6.9 & 7.1 & 6.3 \\
K 3 & 6.8 & 6.2 & 3.4 & 5.4 & 3.9 & 4.5 \\
K 28 & 9.1 & 4.1 & 3.4 & 2.9 & 3.4 & 2.9 \\
\hline
\end{tabular}

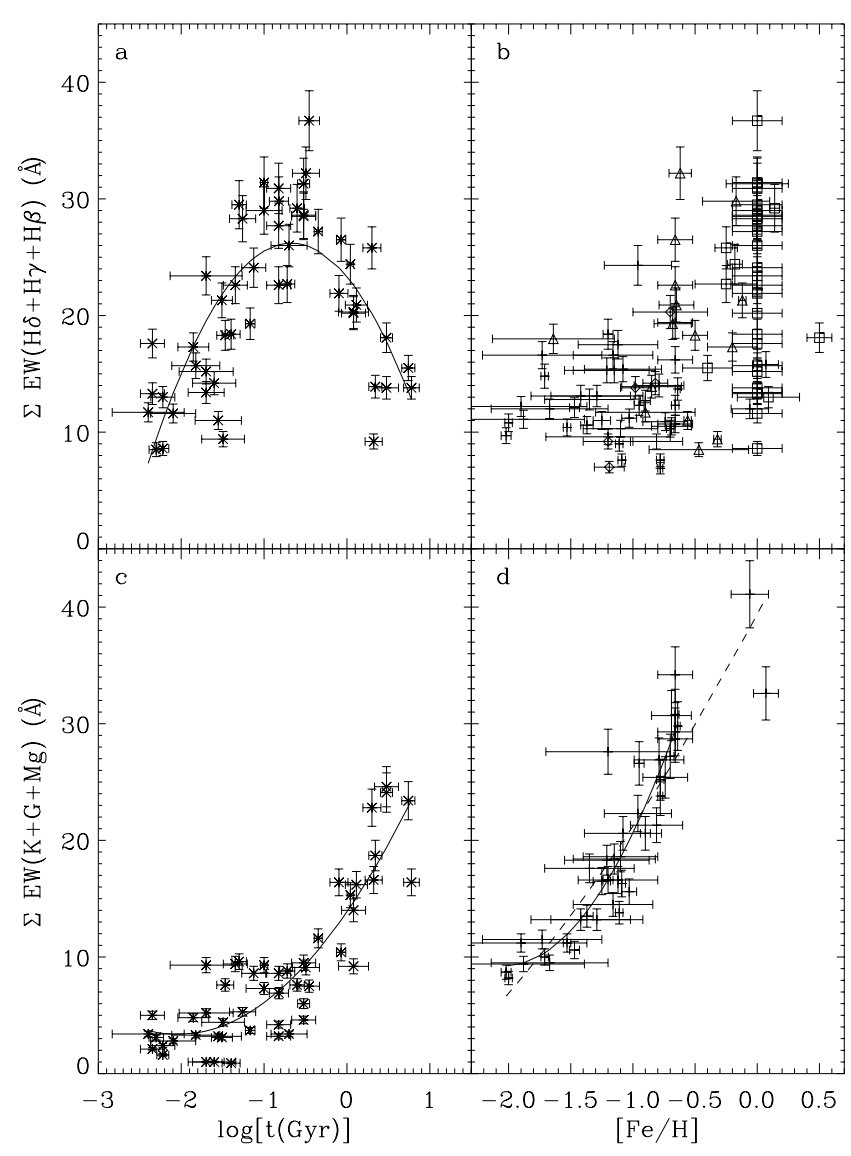

Fig. 2. Same as Fig. 1, including error bars, for selected subsamples: a) Balmer $\mathrm{H}$ equivalent width against age for clusters with $t<10 \mathrm{Gyr}$; a 2nd order polynomial fitted to the data is superimposed; b) Balmer $\mathrm{H} E W$ against $[\mathrm{Fe} / \mathrm{H}]$; c) metallic features $E W$ against age for clusters with $t<10 \mathrm{Gyr}$; a 2nd order polynomial fit is shown; d) metallic features $E W$ against $[\mathrm{Fe} / \mathrm{H}]$ for Galactic globular clusters; the continuous line corresponds to a 2 nd order polynomial fitted to clusters with $[\mathrm{Fe} / \mathrm{H}]<-0.5$, while the dashed line is a similar fit for the whole sample of GGCs.

adjusted a quadratic polynomial given by the expression:

$$
\begin{aligned}
\Sigma E W(\mathrm{H} \delta+\mathrm{H} \gamma+\mathrm{H} \beta)= & k_{1}+k_{2} \cdot \log t(\mathrm{Gyr}) \\
& +k_{3} \cdot(\log t(\mathrm{Gyr}))^{2}
\end{aligned}
$$

where $k_{1}, k_{2}$, and $k_{3}$ resulted to be $23.32 \pm 0.20,-8.56 \pm 0.35$, and $-6.35 \pm 0.18$, respectively. For $\Sigma E W(\mathrm{~K}+\mathrm{G}+\mathrm{Mg})$ versus the cluster age, we fitted the equation:

$$
\begin{aligned}
\Sigma E W(\mathrm{~K}+\mathrm{G}+\mathrm{Mg})= & p_{1}+p_{2} \cdot \log t(\mathrm{Gyr}) \\
& +p_{3} \cdot(\log t(\mathrm{Gyr}))^{2}
\end{aligned}
$$

where $p_{1}, p_{2}$, and $p_{3}$ are $13.88 \pm 0.20,10.32 \pm 0.35$, and $2.53 \pm 0.18$, respectively. The root mean square errors of Eqs. (3) and (4) turned out to be 4.8 and 2.9 , respectively, the equations being valid in the range $-2.4<\log t(\mathrm{Gyr})<0.8$ (see Figs. 2a,c).

The curve fitting shown with solid line in Fig. 2d, i.e., the calibration including GGCs for which $[\mathrm{Fe} / \mathrm{H}]<-0.5$ is given by the equation:

$\Sigma E W(\mathrm{~K}+\mathrm{G}+\mathrm{Mg})=q_{1}+q_{2} \cdot[\mathrm{Fe} / \mathrm{H}]+q_{3} \cdot([\mathrm{Fe} / \mathrm{H}])^{2}$ 
where $q_{1}=53.6 \pm 1.5, q_{2}=43.7 \pm 2.5, q_{3}=10.78 \pm 0.96$ and $\sigma(\Sigma E W(\mathrm{~K}+\mathrm{G}+\mathrm{Mg}))=2.7$. The relationship is valid in the range $-2.0<[\mathrm{Fe} / \mathrm{H}]<-0.65$. If the fit is performed using all GGCs in our sample $(-2.0<[\mathrm{Fe} / \mathrm{H}]<0.07)$, the coefficients for the expression:

$\Sigma E W(\mathrm{~K}+\mathrm{G}+\mathrm{Mg})=u_{1}+u_{2} \cdot[\mathrm{Fe} / \mathrm{H}]+u_{3} \cdot([\mathrm{Fe} / \mathrm{H}])^{2}$

give $u_{1}=39.40 \pm 0.63, u_{2}=20.1 \pm 1.1, u_{3}=1.92 \pm 0.50$, and $\sigma(\Sigma E W(\mathrm{~K}+\mathrm{G}+\mathrm{Mg}))=3.1$.

To facilitate the direct use of Eqs. (4) to (6) we present below the corresponding inverted expressions.

For clusters younger than $\log t(\mathrm{Gyr})<0.8$ :

$\log t(\mathrm{Gyr})=a_{1}+a_{2} \cdot \Sigma E W m+a_{3} \cdot(\Sigma E W m)^{2}$

where $\Sigma E W(\mathrm{~K}+\mathrm{G}+\mathrm{Mg})$ was abbreviated to $\Sigma E W m$ and the coefficients are $a_{1}=-2.18 \pm 0.38, a_{2}=0.188 \pm 0.080, a_{3}=$ $-0.0030 \pm 0.0032$, with $\sigma(\log t(\mathrm{Gyr}))=0.48$.

For GGCs with $[\mathrm{Fe} / \mathrm{H}]<-0.5$ :

$[\mathrm{Fe} / \mathrm{H}]=b_{1}+b_{2} \cdot \Sigma E W m+b_{3} \cdot(\Sigma E W m)^{2}$

with $b_{1}=-2.9 \pm 1.2, b_{2}=0.14 \pm 0.14, b_{3}=-0.0023 \pm 0.0033$, and $\sigma([\mathrm{Fe} / \mathrm{H}])=0.14$.

And for all GGCs:

$[\mathrm{Fe} / \mathrm{H}]=c_{1}+c_{2} \cdot \Sigma E W m+c_{3} \cdot(\Sigma E W m)^{2}$

with $c_{1}=-2.48 \pm 0.98, c_{2}=0.088 \pm 0.097, c_{3}=-0.0008 \pm$ 0.0022 , and $\sigma([\mathrm{Fe} / \mathrm{H}])=0.16$.

\section{Diagnostic diagrams}

In order to provide a useful tool for stellar population studies, we discuss in this section diagrams based on the two used $E W$ sums. After testing several combinations, we built two significant diagrams in terms of discriminating clusters of different ages. Figures 3 and 4 show these diagrams, namely, [ $\Sigma E W(\mathrm{~K}+\mathrm{G}+\mathrm{Mg})-\Sigma E W(\mathrm{H} \delta+\mathrm{H} \gamma+\mathrm{H} \beta)]$ vs. $\quad \Sigma E W(\mathrm{~K}+\mathrm{G}+\mathrm{Mg})$ and $\Sigma E W(\mathrm{~K}+\mathrm{G}+\mathrm{Mg}) \quad$ vs. $\Sigma E W(\mathrm{H} \delta+\mathrm{H} \gamma+\mathrm{H} \beta)$.

Together with the calibrations presented in Fig. 2, these diagnostic diagrams may allow one to estimate ages for Galactic and extragalactic star clusters, and overall metal abundances for globular clusters, using their integrated spectra in the visible range. The behaviour of the $E W$ sums and their combinations with cluster parameters is very different above and below $t \approx 10 \mathrm{Gyr}$. $\Sigma E W(\mathrm{~K}+\mathrm{G}+\mathrm{Mg})$ is well determined by $[\mathrm{Fe} / \mathrm{H}]$ for clusters with $t>10 \mathrm{Gyr}$, being independent of age within this range (see Fig. 2d). For clusters with $t<10 \mathrm{Gyr}$, age is the dominant parameter on both $\Sigma E W(\mathrm{~K}+\mathrm{G}+\mathrm{Mg}$ ) (see Fig. 2c) and $\Sigma E W(\mathrm{H} \delta+\mathrm{H} \gamma+\mathrm{H} \beta)$ (see Fig. 2a).

On the basis that both $E W$ sums are available and measured with an accuracy better than $10 \%$, we propose the following iterative procedure to obtain ages of star clusters: firstly, start using Figs. 3 and 4 to get a first estimate of the cluster age. Secondly, derive calculated $E W$ sums by using Eqs. (3) and (4) with the estimated average age. Thirdly, use these calculated $E W \mathrm{~s}$ values to derive an improved age estimate from

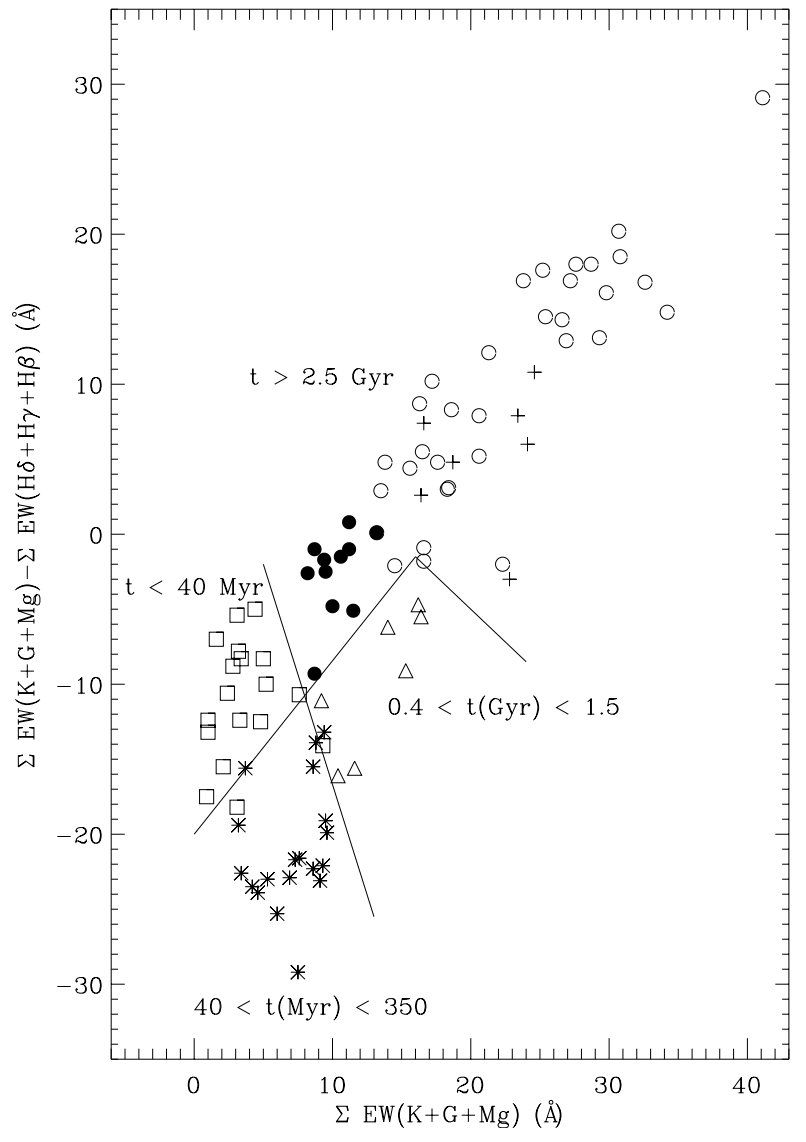

Fig. 3. The difference between $E W$ of metallic features and $E W$ of Balmer $\mathrm{H}$ as a function of $E W$ of metallic features. Different symbols and lines discriminate clusters by age. Galactic globular clusters are indicated by open circles $([\mathrm{Fe} / \mathrm{H}]>-1.4)$ and filled circles $([\mathrm{Fe} / \mathrm{H}] \leq-1.4)$ and intermediate-age clusters $(2.5<t(\mathrm{Gyr})<10)$ are shown as plus signs.

Figs. 3 and 4 . The procedure can be iterated until a fixed difference between the updated and previous $E W \mathrm{~s}$ values is reached. Usually, no more than one iteration is needed to reach a precision of $0.1 \AA$ in $\Sigma E W(\mathrm{H} \delta+\mathrm{H} \gamma+\mathrm{H} \beta)$ and $\Sigma E W(\mathrm{~K}+\mathrm{G}+\mathrm{Mg})$. Note that globular age-like clusters fall outside the calibrating age range. However, globular age-like clusters can be recognized in the diagnostic diagrams. Metal-poor GGCs with $[\mathrm{Fe} / \mathrm{H}] \leq-1.4$, represented by filled circles in Figs. 3 and 4, lie in a distinct region to that occupied by younger clusters. On the other hand, metal-richer globular clusters, represented by open circles in Figs. 3 and 4, are approximately distributed in both figures over the same regions as intermediate-age clusters $(2.5<t(\mathrm{Gyr})<10)$. Consequently, one could be dealing with a metal-rich globular or an intermediate-age cluster without noticing it. For confirmed globular age-like clusters, metallicities can be obtained from the calibrations of Fig. 2d.

\section{Concluding remarks}

We present new calibrations and diagnostic diagrams based on visible integrated spectral features, which will allow one to derive ages for Galactic and extragalactic star clusters, and also overall metallicities for clusters older than 10 Gyr. For that 


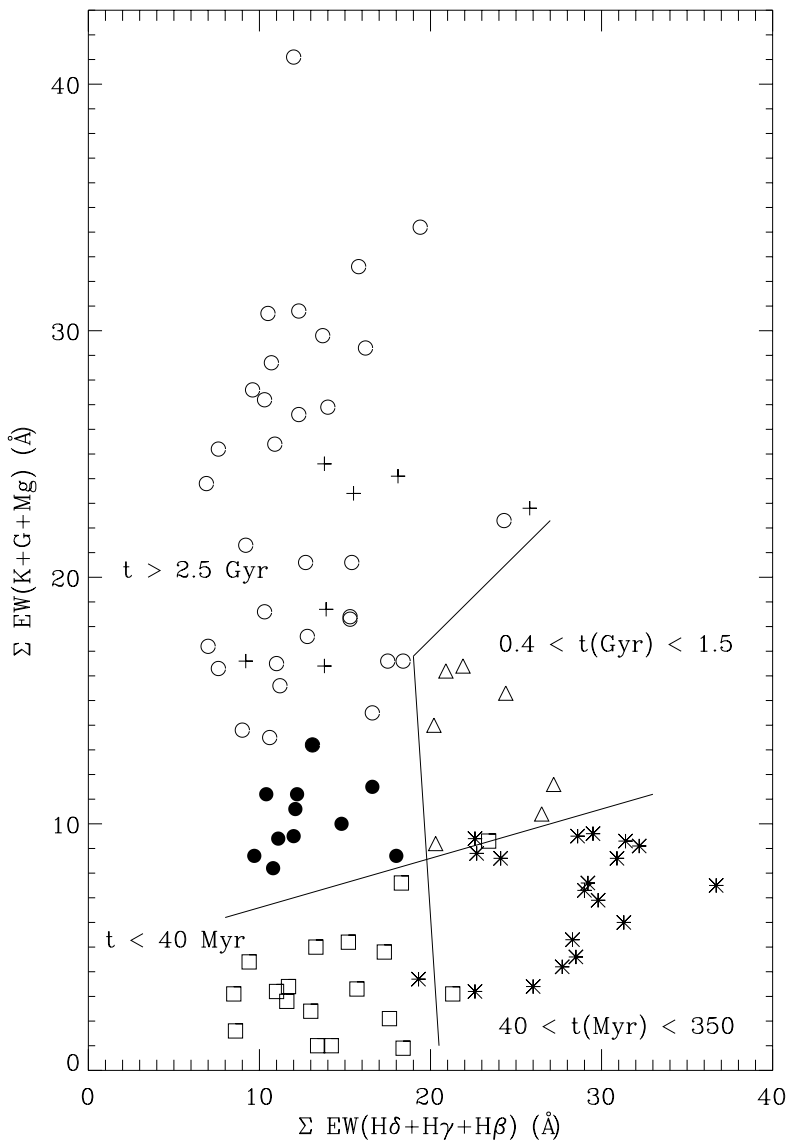

Fig. 4. Sum of metallic versus Balmer $\mathrm{H} E W$ s. Symbols and lines are as in Fig. 3.

purpose, we first searched the literature for star clusters with integrated spectra and reliable determinations of their ages and metallicities. This task led us to compile the largest sample of star clusters, covering wide ranges of age and metallicity and with the required integrated spectra available. We carried out an analysis as carefully as possible of ages and metal abundances of each selected object, in order to put them into homogeneous scales. As far as we are aware, this is the first time that such a sample of star clusters with homogeneous ages and metallicities is provided.

Using these homogeneous scales, we investigated the behaviours of different integrated spectral indices as a function of cluster age and metallicity. The spectral indices were built on the basis of the measurements of $E W \mathrm{~s}$ of $\mathrm{K} \mathrm{Ca} \mathrm{II,} \mathrm{G} \mathrm{band}(\mathrm{CH})$ and $\mathrm{MgI}$ metallic lines, and $\mathrm{H} \delta, \mathrm{H} \gamma$, and $\mathrm{H} \beta$ Balmer lines. The fact that all the $E W \mathrm{~s}$ have been measured following the same precepts also guarantees homogeneity within the data. From the analysis, we found that both sums of the metallic and Balmer $\mathrm{H}$ line $E W \mathrm{~s}$, separately, are good indicators of cluster ages for objects younger than $10 \mathrm{Gyr}$. Likewise, $\Sigma E W(\mathrm{~K}+\mathrm{G}+\mathrm{Mg})$ is useful for metallicity determinations of star clusters older than 10 Gyr. The sensitivity of the suggested integrated spectral indices to cluster age or metallicity in the respective age domain does not appear to be degenerated by the counterpart parameter, in the sense that the $E W$ sum, which is an age indicator, is not a metallicity index in the corresponding age range, and vice versa.

Finally, we propose an iterative procedure for estimating star cluster ages from two new diagnostic diagrams and two calibrations in terms of the mentioned $E W$ sums. The method allows one to estimate cluster ages with an internal precision better than $10 \%$. For globular age-like clusters, we obtained a calibration of $\Sigma E W(\mathrm{~K}+\mathrm{G}+\mathrm{Mg})$ as a function of the ironto-hydrogen ratio. We foresee that this technique will serve to estimate ages and metallicities of relatively faint clusters now reacheable with the new generation of telescopes and instruments.

Acknowledgements. We thank the referee, Dr. Raffaele G. Gratton, for helping to improve this paper. This work was partially supported by the Argentinian institutions CONICET, and Agencia Nacional de Promoción Científica y Tecnológica (ANPCyT).

\section{References}

Anthony-Twarog, B. J., \& Twarog, B. A. 2000, AJ, 120, 3111

Armandroff, T. E., \& Zinn, R. 1988, AJ, 96, 92

Armandroff, T. E., \& Da Costa, G. S. 1991, AJ, 101, 1329

Beasley, M. A., Hoyle, F., \& Sharples, R. M. 2002, MNRAS, 336, 168

Bica, E. 1988, A\&A, 195, 76

Bica, E., \& Alloin, D. 1986a, A\&A, 162, 21

Bica, E., \& Alloin, D. 1986b, A\&AS, 66, 171

Bica, E., Alloin, D., \& Santos Jr., J. F. C. 1990, A\&A, 235, 103

Bica, E., Clariá, J. J., Dottori, H., Santos Jr., J. F. C., \& Piatti, A. E. 1996, ApJS, 102, 57

Cameron, L. M. 1985, A\&A, 146, 59

Carraro, G., Girardi, L., \& Marigo, P. 2002, MNRAS, 332, 705

Carretta, E., \& Gratton, R. G. 1997, A\&AS, 121, 95 (CG97)

Carretta, E., Cohen, J. G., Gratton, R. G., \& Behr, B. B. 2001, AJ, 122, 1469

Carretta, E., Gratton, R. G., Clementini, G., \& Fusi Pecci, F. 2000, ApJ, 533, 215

Cohen, J. 1982, ApJ, 258, 143

Cohen, J. G., Gratton, R. G., Behr, B. B., \& Carretta, E. 1999, ApJ, 523, 739

Da Costa, G. S., \& Hatzidimitriou, D. 1998, AJ, 115, 1934

de Grijs, R., Gilmore, G. F., Johnson, R. A., \& Mackey, A. D. 2002, MNRAS, 331, 245

Dirsch, B., Richtler, T., Gieren, W. P., \& Hilker, M. 2000, A\&A, 360, 133

Dutra, C. M., Bica, E., Clariá, J. J., \& Piatti, A. E. 1999, MNRAS, 305, 373

Elson, R. A. W., \& Fall, S. M. 1985, ApJ, 299, 211

Friel, E. D., Janes, K. A., Tavarez, M., et al. 2002, AJ, 124, 2693

Geisler, D., Bica, E., Dottori, H., et al. 1997, AJ, 114, 1920

Geisler, D., \& Sarajedini, A. 1999, AJ, 117, 308

Girardi, L., Chiosi, C., Bertelli, G., \& Bressan, A. 1995, A\&A, 298, 87

Harris, W. E. 1996, AJ, 112, 1487

Heasley, J. N., Janes, K. A., Zinn, R., et al. 2000, AJ, 120, 879

Hill, V., François, P., Spite, M., Primas, F., \& Spite, F. 2000, A\&A, 364, 19

Jasniewicz, G., \& Thévenin, F. 1994, A\&A, 282, 717

Johnson, R. A., Beaulieu, S. F., Gilmore, G. F., et al. 2001, MNRAS, 324,367

Johnson, J. A., Bolte, M., Stetson, P. B., Hesser, J. E., \& Somerville, R. S. 1999, ApJ, 527, 199 
Leonardi, A. J., \& Rose, J. A. 2003, AJ, 126, 1811

Mighell, K. J., Sarajedini, A., \& French, R. S. 1998, AJ, 116, 2395

Minniti, D. 1995, A\&A, 303, 468

Oliva, E., \& Origlia, L. 1998, A\&A, 332, 46

Olsen, K. A. G., Hodge, P. W., Mateo, M., et al. 1998, MNRAS, 300, 665

Olszewski, E. W., Schommer, R. A., Suntzeff, N. B., \& Harris, H. C. 1991, AJ, 101, 515

Phelps, R. L., \& Schick, M. 2003, AJ, 126, 265

Piatti, A. E., Bica, E., Clariá, J. J., Santos Jr., J. F. C., \& Ahumada A. V. 2002a, MNRAS, 335, 233

Piatti, A. E., Sarajedini, A., Geisler, D., Bica, E., \& Clariá, J. J. 2002b, MNRAS, 329, 556

Piatti, A. E., Santos Jr., J. F. C., Clariá, J. J., et al. 2001, MNRAS, 325, 792

Rabin, D. 1982, ApJ, 261, 85

Rosenberg, A., Saviane, I., Piotto, G., \& Aparicio, A. 1999, AJ, 118, 2306

Rutledge, G. A., Hesser, J. E., \& Stetson, P. B. 1997, PASP, 109, 907 (R97)
Santos Jr., J. F. C., Alloin, D., Bica, E., \& Bonatto, C. 2002, in IAU Symp., 207, ed. D. Geisler, E. K. Grebel, \& D. Minniti (San Francisco: Astronomical Society of the Pacific), 727

Santos Jr., J. F. C., \& Bica, E. 1993, MNRAS, 260, 915

Santos Jr., J. F. C., Bica, E., Clariá, J. J., et al. 1995, MNRAS, 276, 1155

Searle, L., Wilkinson, A., \& Bagnuolo, W. G. 1980, ApJ, 239, 803

Suntzeff, N. B., Schommer, R. A., Olszewski, E. W., \& Walker, A. R. 1992, AJ, 104, 1743

Tadross, A. L. 2003, New Astron., 8, 737

Testa, V., Ferraro, F. R., Chieffi, A., et al. 1999, AJ, 118, 2839

Twarog, B. A., Anthony-Twarog, B. J., \& de Lee, N. 2003, AJ, 125, 1383

Twarog, B. A., Ashman, K. M., \& Anthony-Twarog, B. J. 1997, AJ, 114,2556

West, M. J., Côté, P., Marzke, R. O., \& Jordán, A. 2004, Nature, 427, 31

Zinn, R., \& West, M. J. 1984, ApJS, 55, 45 (ZW84) 